\title{
The CMS Object-Oriented Simulation
}

S. Abdoulline, FNAL, USA, N. Amapane, University of Torino, Italy, F. Ambroglini, INFN, Perugia, Italy, P. Arce, CIEMAT, Madrid, Spain, S. Ashby, CERN, Geneva, Switzerland,

S. Banerjee, Tata Institute, Mumbai, India, U. Berthon, , IN2P3-CNRS, Palaiseau, France,

T. Boccali, SNS, Pisa, Italy, M. Case, UC Davis, USA, C. Charlot, IN2P3-CNRS, Palaiseau, France,

H. Cheung, FNAL, USA, T. Cox, UC Davis, USA,

N. Darmenov, INRNE, Sofia, Bulgaria and CERN, Geneva, Switzerland, A. De

Roeck, CERN, Geneva, Switzerland, X. Ding, Nanjing University, China, S. Dutta, University of Pisa, Italy,

D. Elvira, FNAL, USA, F. Ferro, INFN, Genova, Italy, A. Giammanco, INFN, Pisa, Italy,

P. Govoni, INFN Milano-Bicocca, Italy, O. Gutsche, FNAL, USA,

$\mathrm{X}$. Huang, University of Puerto Rico (Mayaguez), USA, V. Innocente, CERN, Geneva, Switzerland,

C. Jones, Cornell University, Ithaca, NY, USA, A. Kharchilava, SUNY at Buffalo, USA,

P. Katsas, University of Athens, Greece, J. Kowalkowski, FNAL, USA, K. Lassila-Perini, HIP, Helsinki, Finland, S. Magni, INFN Milano-Bicocca, Italy, P. Meridiani, INFN, Roma, Italy,

F. Moortgat, CERN, Geneva, Switzerland, A. Nikitenko, Imperial College, London, UK,

I. Osborne, NorthEastern University, USA, M. Paterno, FNAL, USA, M. Pioppi, INFN Perugia, Italy,

S. Piperov, INRNE, Sofia, Bulgaria and CERN, Geneva, Switzerland, N. Ratnokiva, FNAL, USA,

A. Rizzi, SNS, Pisa, Italy, E. Sexton-Kennedy, FNAL, USA, E. Shabalina, University of Illinois, Chicago, USA,

L. Silvestris, INFN, Bari, Italy, M. Spiropulu, CERN, Geneva, Switzerland, M. Stavrianakou, FNAL, USA,

W. Tanenbaum, FNAL, USA, J. Weng, Karlsruhe University, Germany and CERN, Geneva, Switzerland,

T. Wildish, Princeton University, USA, R. Wilkinson, California Inst. of Tech., Pasadena, USA,

S. Wynhoff, Princeton University, USA, A. Yagil, FNAL, USA, J. Yarba, FNAL, USA, M. Zanetti, INFN, Italy,

A. Zhokin, ITEP, Moscow, Russia

\begin{abstract}
The CMS Object Oriented Geant4-based program is used to simulate the complete central CMS detector (over 1 million geometrical volumes) and the forward systems such as the Totem telescopes, Castor calorimeter, Zero Degree Calorimeter, Roman Pots, and the Luminosity Monitor. The simulation utilizes the full set of electromagnetic and hadronic physics processes provided by Geant 4 and detailed particle tracking in the 4 Tesla magnetic field. Electromagnetic shower parameterization can be used instead of full tracking of high-energy electrons and positrons, allowing significant gains in speed without detrimental precision losses. The simulation physics has been validated by comparisons with test beam data and previous simulation results. The system has been in production for almost two years and has delivered over 100 million events for various LHC physics channels. Productions are run on the US and EU grids at a rate of 3-5 million events per month. At the same time, the simulation has evolved to fulfill emerging requirements for new physics simulations, including very large Heavy Ion events and a variety of SUSY scenarios. The software has also undergone major technical upgrades. The framework and core services have been ported to the new CMS offline software architecture and Event Data Model. In parallel, the program is subjected to ever more stringent quality assurance procedures, including a recently commissioned automated physics validation suite.
\end{abstract}

\section{INTRODUCTION}

The CMS simulation suite manages all CMS detectors, both central (Tracker, Calorimeters and Muons), Fig. 1, and forward (CASTOR calorimeter, Totem telescopes, the Zero Degree Calorimeter (ZDC), the Roman Pots and the Luminosity Monitor), as well as several test beam layouts and prototypes. It implements their sensitive detector behaviour, track selection mechanisms, hit collections and digitization.

The system is based on the Geant4 [1] toolkit and the CMS object-oriented framework and event data model.

The standard application with the central systems in the CMS 4 Tesla magnetic field, and physics processes simulated with the Geant 4 physics list, has been extensively validated in terms of physics and is regularly tested following the evolution of Geant 4 and the CMS software framework.

\section{Simulation ARCHITECTURE AND FRAMEWORK}

As mentioned above, the simulation is based on the CMS framework and event data model. This ensures overall consistency and usability, minimizes overheads from development and support of non simulation-specific elements and facilitates maintenance and quality assurance.

Given the vast scope of LHC detector and physics simulation, extensibility and configurability are fundamental prerequisites. This is naturally supported by the concept of an event processing module, which encapsulates a unit of clearly defined eventprocessing functionality, allowing independent development and verification. 


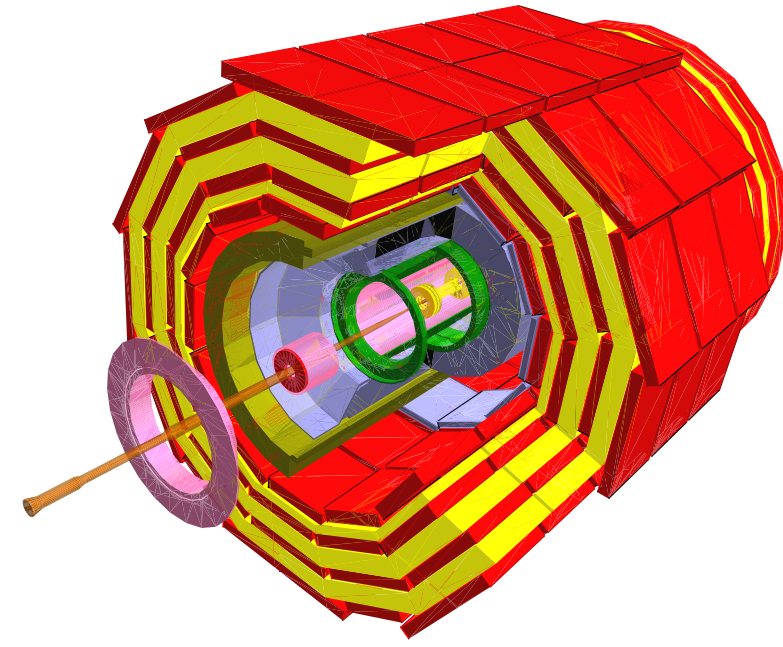

Fig. 1. The CMS central detector

The framework ensures reproducibility by enforcing immutability for the event products and by automatically recording the provenance for each event processing module. The natural persistent form of the event data is a Root tree, which allows straightforward access for monitoring and analysis.

The framework EventProcessor handles the initialization and event processing loop. The EventProcessor parses the configuration information provided by the user, creates the unscheduled framework modules which are listed in the configuration and then uses the ScheduleBuilder to create the scheduled modules, the ScheduleExecutor to run the sequence of Event-based modules and the EventSetupProvider to manage all the nonEvent data services. Each Module instance is configured with a ParameterSet. Modules must not interact directly with other modules. Internal algorithms are configured by percolating ParameterSets to the algorithm, by the Module that contains the algorithm. The elements that compose the event data stored in an Event are called EDProducts. There is no actual class EDProduct; rather, EDProduct is a generic programming concept. An object of almost any type provided it is defaultconstructible, copyable and destructible, and can be made persistent, may be used as an EDProduct.

The event-processing chain may be cut and the state of the event saved between any two modules. This facilitates combinations of simulated hit formatting, with or without subsequent event mixing (pile-up) and digitization in the context of a single simulation application.

On-demand processing, referred to as unscheduled as opposed to the scheduled scheme described above, is a key simulation requirement. An unscheduled application is configured by specifying a selection of independent top-level EDProducts to be written out, or a selection of independent modules to be run, or some combination of the above, as well as the menu of ED-
Producers that should be known to the EDProducer registry. A signaling mechanism, based on the dispatcher-observer pattern, may be used to trigger an unscheduled application component, such as a user monitoring action.

For the detector description the simulation utilizes the CMS Detector Description services and the DDD/XML description files from the CMS Geometry packages. Visualization and event display are handled by separate visualization packages, interfaced to the core and detector-specific simulation systems.

\section{Core Application, Run and Event Management}

The application core consists of the framework-based Event Data Producer (EDProducer) module and the Geant4-based RunManager. The former holds the declarations of the Event Data produced during the simulation and is responsible for the initialization, event processing loop and termination of the simulation application. The data are fetched by the RunManager and are stored as Event Data Products (EDProducts) in the Event held by the EDProducer.

The RunManager instantiates a G4RunManagerKernel and controls standard interfaces to Geant4 for the event generation, physics lists, as well as the interfaces to the run, event, stacking, tracking and stepping actions. The RunManager handles the storage and retrieval of the cross-section tables as built for a given detector configuration and physics list. Reading the prebuilt cross-section tables at the beginning of an application can reduce the overall initialization time by as much as a factor of 4 . The RunManager also handles the storage and retrieval of run and event random number seeds. This feature facilitates debugging of rare crashes in time-consuming physics events.

\section{INFRASTRUCTURE AND SERVICES}

Detector geometry construction is automated: the EventSetup accesses the detector description record (for the moment, "ideal" geometry only, although inclusion of misalignment is also foreseen) and passes it to the geometry builder which converts Detector Description solids and materials to their Geant4 counterparts as well as the logical and physical volumes needed to build the Geant 4 geometry for the chosen configuration. The Detector Description SpecPars mechanism allows the definition of special parameter sets (extra attributes, field parameters, range cuts etc) to be associated with selected detectors, as needed by specific applications.

Magnetic field services are provided by the independent CMS MagneticField subsystem, interfaced to the simulation infrastructure, so as to allow choice of field type and configuration of stepping and propagation parameters.

Monte Carlo event generation is provided by the CMS IOMC subsystem. The Monte Carlo events, in the HepMC format, may be produced and read on the fly (typical for the so-called particle guns) or pregenerated and read from a POOL database or ASCII files. The primary events are converted to Geant 4 events for subsequent simulation in the CMS detector. The conversion method creates a primary (collision) vertex with the generated coordinates and assigns to it the primary particles that 
survive acceptance cuts. The primary event vertex, the proper decay times of the unstable particles and their predefined decay products are used by Geant 4 to create the secondary vertices after correctly propagating and simulating the unstable particles until they decay as predefined.

The final Monte Carlo truth record contains the generated event particles with their tracks, vertices and decay trees from the original generator event, as well as selected tracks from the Gean4 simulation. Records for the latter are assembled by the EventAction and TrackingAction and are organized so as to allow navigation from hits to their corresponding tracks and parent vertices. Simulated tracks are stored if they have been flagged for saving at various points (tracking, stepping, hit processing etc) of the actual CMS simulation. These tracks are selected either because they have produced hits in the sensitive detectors or because they have been identified as important for the interaction history and the eventual reconstruction of the full tree.

The infrastructure for physics lists and production cuts allows the implementation of several kinds of physics lists with extensive run-time configurability: choice of physics subset (propagation only for debugging and material studies, standalone electromagnetic etc), choice of hadronic physics model (LHEP, QGSP, QGSC and FTFP), optional use of parameterized electromagnetic shower simulation with the GFlash package and optional activation and tailoring of individual processes such as synchrotron radiation and gamma/e-nuclear interactions.

\section{Hit COLLECtion, EVENT MiXING (PILE-UP) AND DIGITIZATION}

Common data formats for the final hit and digit EDProducts are used for the two major types of detectors for tracking and calorimetry. Hit processing and collection are handled by the detector subsystems, with interfaces to the Geant 4 methods invoked at the stepping and end of event actions.

During the "low luminosity" and "high luminosity" phases of its operation, the LHC machine will produce an average of respectively 3.5 and 17.5 minimum bias pile-up interactions per bunch crossing on top of the trigger event. In addition to this in-time pile-up, it is necessary to account in the simulation for out-of-time pile-up, i.e. for pile-up coming from bunch crossings before and after the trigger event crossing, with a number of crossings to consider before and after the nominal one depending on the front end time response of the different subdetectors. Special conditions as crossings with no pile-up before and crossings with a number of following crossings without pile-up are also considered.

As the pile-up addition depends on the machine luminosity and conditions and is much less time consuming than the detector simulation, the pile-up events are simulated separately from the physics events and the simulation outputs are merged according to the desired luminosity in an independent production step. It is therefore possible to reuse physics simulated events to be merged with pile-up events for a different machine luminosity. In order to avoid introducing too many correlations and biases, a sample of $500 \mathrm{~K}$ pile-up events is used and the reuse of the same pile-up event in a given sequence is forbidden, while the simulated pile-up events passing trigger requirements are filtered out.

The mixing module is in charge of producing the merged event. It is a framework module whose task is to merge events from a primary stream with a number of events from a secondary stream. The product (EDProduct) of this module is a CrossingFrame, which in turn is the input for the digitization.

Digitization, which consists in the simulation of the electronic readouts used to acquire data in the DAQ system, starts from the positions and simulated energy losses in the sensitive detectors, and produces an output as close as possible to the real data from CMS, plus the additional Monte Carlo truth information available in the simulation production.

In tracking detectors (strips and pixels), the energy loss distributions along the modules, including Landau fluctuations, drift and diffusion, as well as noise and couplings between channels are taken into account.

For the electromagnetic calorimeter digitization, where the active volumes are crystals and silicon strips, the energy deposition and hit arrival time are recorded, taking into account the light collection efficiency for the crystals.

In the hadronic calorimeter digitization, energy deposition in the scintillators is converted to number of photoelectrons, taking into account fluctuations and noise, and then depositions from up to 5 previous and 3 subsequent crossings are added.

In the Muon Drift Tube (MDT) system, particular care is taken in simulating the behavior of the drift cells as a function of the muon direction and impact position with respect to the sense wire, and of the residual magnetic field effects. The resulting drift time is smeared so as to obtain a 4 ns resolution, corresponding to an intrinsic cell resolution of about 220 microns, as measured in test beam data. The output signal for the hit reconstruction is obtained by adding the muon timeof-flight from the collision vertex and the propagation time of the signal along the cell wire.

The digitization step of the Cathode Strip Chamber (CSC) system involves simulating the responses of the ADCs and discriminators connected to the strips and wires. To create the analog signals seen by the CSC wire and strip electronics, parameterizations of the amplifier and shaper response are convoluted with the ion drift collection time. Cross-talk, noise and readout dead time (200 ns) are taken into account before the storage of the strip signals in Switched Capacitor Arrays (SCA) is simulated and the signal shape is sampled and stored.

The Resistive Plate Chamber (RPC) response is assumed to take place within $20 \mathrm{~ns}$ of the passage of a charged particle through the detector with a 3 ns Gaussian jitter, which also accounts for the contribution from the front-end electronics and the cables to the link board.

\section{Simulation VAlidation AND PHYSiCS STUdies}

High energy physics experiments depend critically on the accuracy of physics generators and detector simulations. Simulated data events are used for detector design optimization, 
calibration, object identification, and physics analysis. The size of systematic uncertainties associated with particle discoveries, mass, or cross section measurements is tightly associated with how accurately the simulations describe the actual performance of the detector in measuring electrons, photons, and hadrons. It is essential, for the success of a HEP experiment, to understand and tune the physics of the simulation tool to agree with the data measurements. Although not always explicitly mentioned, the Geant4 simulation results described in the subdetector sections are compared with test beam data, wherever available, and results from the Geant3-based program.

\section{A. Tracker simulation and validation}

Tracker simulation has played a key role in the development and optimization of the simulation infrastructure and the validation process. The tracker material budget, which can only be correctly estimated with a very detailed description of all active and passive detector components, directly affects the electromagnetic calorimeter physics performance and places stringent requirements on the accuracy of the detector description and geometry construction. A navigable Monte Carlo truth, for correct decay tree reconstructions, as well as the proper treatment of hard electron bremsstrahlung are of vital importance in B- $\tau$ studies, in which the tracker plays a key role. With the above requirements satisfied, tracker performance has been extensively validated in terms of tracking and hit distributions for single particles, minimum bias, and physics events.

\section{B. Electromagnetic calorimeter (ECAL) simulation and valida- tion}

Initial studies based on a comparison between a Geant4based simulation and test beam data provide evidence that Geant4 gives an excellent representation of electromagnetic showers. Overall ECAL performance, in terms of energy and position resolution, is dominated by effects that are not part of the shower simulation, such as electronics noise, photostatistics, longitudinal uniformity of light collection, and crystal intercalibration. These effects are taken into account in the digitization step of the simulation process. For this reason, only gross errors are identified by a comparison of energy and position resolution taken from Geant 4 shower information. On the other hand, the largest sensitivity is to changes or errors in the radiation and showering in the tracker material. Unfortunately the accurate simulation of this effect cannot be validated in the test beam. The shower lateral distribution, and its fluctuations from shower to shower is an important quantity which can be validated comparing the Monte Carlo simulations with test beam measurements. In particular, parameters sensitive to the lateral shower shape, which affects the fraction of incident energy contained in ECAL clusters, are measured in the test beam.

\section{Hadronic calorimeter $(H C A L)$ simulation and validation}

HCAL studies on energy resolution and linearity, e/ $\pi$ ratio, Fig. 2, and shower profile are instrumental in Geant 4 hadronic physics validation, in the context of the LCG simulation physics validation project. They are based on comparisons between single particle measurements in test beam experiments and Geant 4 based simulations of the associated detector setup. In 20022004, several HCAL test beam experiments exposed different HCAL modules, preceded by an electromagnetic calorimeter prototype, to beams of pions, electrons and muons over a large energy range. The data were compared with Geant4 simulations using the hadronic physics parametric (LHEP) and microscopic (QGSP) models. The pion energy resolution and response linearity as a function of incident energy derived from the simulations are in good agreement with the data within the large systematic uncertainties in the latter. Transverse and longitudinal shower profiles were studied in the 1996 and 2004 test beam experiments. Pion showers predicted by Geant 4 are narrower than those predicted by Geant3. Showers predicted by the QGSP physics list (version 2.7) are shorter than those predicted by the LHEP (version 3.6) list, with LHEP predictions being closer to those from Geant3/Geisha. More precise test beam measurements will hopefully allow to tune the default QGSP physics list to the data.

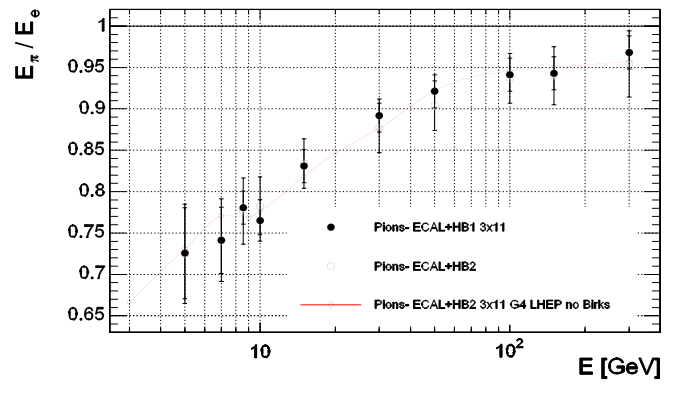

Fig. 2. e/ $\pi$ ratio as a function of energy with test beam 2004 data

\section{Muon system simulation and validation}

Single muons with momenta in the $10 \mathrm{GeV}-10 \mathrm{TeV}$ range have been simulated in the CMS detector using the Geant 3 and Geant4 packages. While ionization is modeled very similarly in both packages, muon bremsstrahlung, $e^{+} e^{-}$production, and in particular, muon-nuclear interaction are significantly different, due to newer theoretical developments included in Geant4. Multiple scattering is significantly smaller in Geant4, in agreement with experimental results. Geant 4 results also show an improvement with respect to Geant 3 in the precision of the propagation of the muons along the detector. The production thresholds on secondary particles in the different regions of the detector were set to a large value to increase performance, but not as large as to kill the particles originated in a passive 
region with enough energy to reach the sensitive detectors. The production of hits in the simulation of the muon system was tested by comparing the Monte Carlo predictions with test beam data. The test beam experiment consisted of two muon chambers with and without an iron slab in between them, to investigate the effect of the muon showers in the passive material. The analysis, based on muons in the 50$300 \mathrm{GeV} p_{T}$ range, show that Geant 4 slightly underestimates soft delta ray production in cell volumes, while hard delta rays and electromagnetic showers are correctly modeled. In spite of this discrepancy, local track reconstruction efficiency and resolution is well reproduced by the simulation.

\section{E. Forward detector simulation and validation}

The forward detectors, such as the CASTOR and ZDC calorimeters, and the Totem telescopes are essential tools for the diffractive and heavy ion programs. For example, the ZDC is a Cerenkov detector designed to collect any remaining neutral fragments of the colliding nuclei and may be used as a measure of the collision centrality. In pp collisions, the ZDC may be incorporated in the study of forward physics and photon production. Simulation studies are underway to study issues such as energy resolution and energy leakage. New test beam data will allow for a more systematic validation of the simulation results. Current validation efforts involve the implementation of a RHIC-design ZDC to take advantage of existing test beam data.

\section{F. Parameterized electromagnetic shower simulation}

The detailed simulation of electromagnetic showers is computationally intensive. A parameterization of the spatial energy distribution of an electromagnetic shower, based on probability density functions [3], allows to speed up the process without compromising the simulation accuracy. In CMS, GFlash is used to parameterize electrons and positrons in the barrel and endcap electromagnetic calorimeter. Comparisons between the GFlash based and the full simulation of the energy depositions in the central crystal, and $3 \times 3,5 \times 5$ crystal matrices show good agreement to the $>1 \%$ level, Fig. 3. Transverse and longitudinal shower profiles are also well modeled by GFlash to within 1-3\%. The GFlash shower parameterizations allow a time performance gain of a factor of 3-10 in the simulation. The gain in speed depends on the event type, the particle energy and the detector region. For instance, a single electron or photon with an energy of $100 \mathrm{GeV}$ in the ECAL barrel is simulated 10 times faster using GFlash. For a large extra dimensions full signal event, $p p \rightarrow \gamma+G$, with a single photon above $1000 \mathrm{GeV}$, the gain in speed is a factor of 4 .

\section{Simulation PERFORMANCE AND PRODUCTIONS}

The simulation suite went into production for the CMS physics community in November 2003. A total of over 100 million events have been simulated by the CMS production team. Most of these events are physics channels, contributing

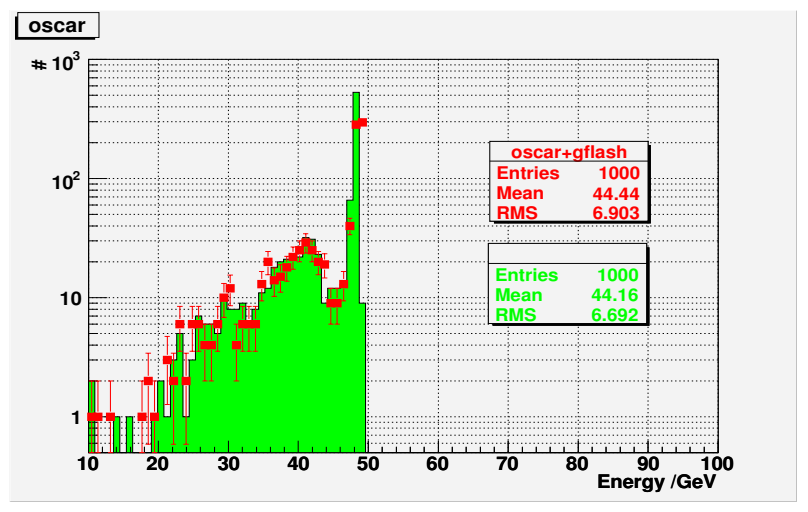

Fig. 3. Energy depositions in a $5 \times 5$ crystal matrix for $50 \mathrm{GeV}$ electrons. (histogram - full Geant4 simulation, red markers - shower parameterization)

to the CMS Physics Technical Design Report analyses. The simulation has proven to be very stable, with a failure rate of around 1 in $10 \mathrm{~K}-10 \mathrm{M}$ events. A typical signal event takes $250 \mathrm{MB}$ of memory, about $100-200 \mathrm{~K} \mathrm{Si} 2 \mathrm{~K}$ of CPU, and produces $1 \mathrm{MB}$ of output data.

\section{SUMMARY AND CONCLUSIONS}

In CMS, the OO simulation suite based on the Geant4 toolkit, has successfully replaced its FORTRAN/Geant3 predecessor. It has been validated and adopted by all CMS detector and physics groups. It has proven robust and performant, easily extensible and configurable.

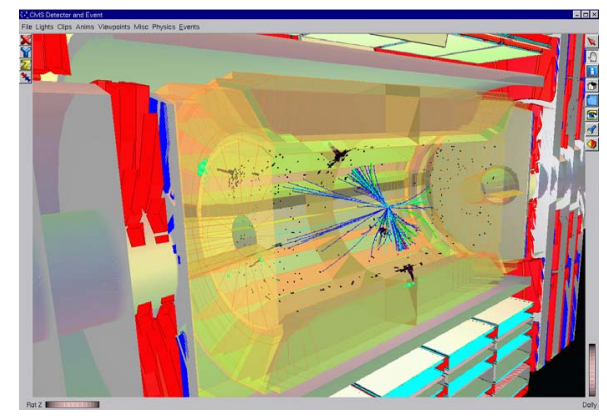

Fig. 4. Black Hole Event in CMS simulation, Model of Landsberg and Dimopoulos, Planck scale $=1 \mathrm{TeV}$, Number of extra dimensions $=2$

It has also proven modular and portable to a different software framework without loss of functionality or usability. New features, extending functionality, such as black hole, Fig. 4, and exotics simulation, or improving performance, such as hadron shower parameterization, the latter envisaged for 2006, can be added without disrupting standard operations and performance.

\section{REFERENCES}

[1] S. Agostinelli et al., "Geant4: a simulation toolkit", NIM A 506 (2003), 250-303

[2] R. Brun et al., "GEANT3 Users Guide", CERN Program Library W5013.

[3] hep-ex/0001020, G. Grindhammer and S. Peters 